\title{
Reappraisal of Supraorbital Sensory Nerve Conduction Recordings: Orthodromic and Antidromic Techniques
}

\author{
Hyeun Jun Park, MD, Sung-Hoon Kim, MD, Se Kwang Lee, MD, \\ Hang Jae Lee, MD, PhD, Hee Kyu Kwon, MD, PhD
}

Department of Physical Medicine and Rehabilitation, Korea University School of Medicine, Seoul, Korea

Objective To establish a supraorbital nerve sensory conduction recording method and assess its usefulness. Methods Thirty-one healthy subjects without a history of trauma or neurological disease were recruited. For the orthodromic procedure, the recording electrode was attached immediately superior to the supraorbital notch. The stimulation electrode was placed on points along the hairline which evoked the largest sensory nerve action potentials (SNAPs). The antidromic sensory response was recorded after switching the recording and stimulating electrodes. The measured parameters were onset latency, peak latency, and baseline to peak amplitude of the SNAPs. The electrophysiological parameters of the bilateral supraorbital nerves were compared. We also recruited two patients who had sensory deficits on one side of their foreheads because of laceration injuries.

Results The parameters of orthodromically recorded SNAPs were as follows: onset latency $1.21 \pm 0.22 \mathrm{~ms}$ (range, 0.9-1.6 ms), peak latency $1.54 \pm 0.23 \mathrm{~ms}$ (range, 1.2-2.2 ms), and baseline to peak amplitude $4.16 \pm 1.92 \mu \mathrm{V}$ (range, 1.4-10 $\mu \mathrm{V}$ ). Those of antidromically recorded SNAPs were onset latency $1.31 \pm 0.27 \mathrm{~ms}$ (range, 0.8-1.7 ms), peak latency $1.62 \pm 0.29 \mathrm{~ms}$ (range, 1.3-2.2 ms), and baseline to peak amplitude $4.00 \pm 1.89 \mu \mathrm{V}$ (range, 1.5-9.0 $\mu \mathrm{V}$ ). There was no statistical difference in onset latency, peak latency, or baseline to peak amplitude between the responses obtained using the orthodromic and antidromic methods, and the parameters also revealed no statistical difference between the supraorbital nerves on both sides.

Conclusion We have successfully recorded supraorbital SNAPs. This conduction technique could be quite useful in evaluating patients with supraorbital nerve lesions.

Keywords Supraorbital nerve, Sensory conduction, Electromyography

Received April 30, 2015; Accepted July 20, 2015

Corresponding author: Hee Kyu Kwon

Department of Physical Medicine and Rehabilitation, Korea University Anam Hospital, Korea University School of Medicine, 73 Inchon-ro, Seongbukgu, Seoul 02841, Korea

Tel: +82-2-920-6471, Fax: +82-2-929-9951, E-mail: hkkwon@korea.ac.kr

(c) This is an open-access article distributed under the terms of the Creative Commons Attribution Non-Commercial License (http://creativecommons.org/ licenses/by-nc/4.0) which permits unrestricted noncommercial use, distribution, and reproduction in any medium, provided the original work is properly cited. Copyright ( 2016 by Korean Academy of Rehabilitation Medicine 


\section{INTRODUCTION}

The supraorbital nerve, which is a terminal branch of the trigeminal nerve, innervates the skin of the forehead and frontoparietal scalp. Therefore, damage to the supraorbital nerve can result in paresthesia or hypoesthesia of the skin that it innervates.

Supraorbital neuropathy can easily result from trauma to the forehead or orbital rim. Supraorbital nerve injury after trauma can lead to post-traumatic supraorbital neuralgia [1]. Injuries can also result from complications of various surgeries such as forehead lifts, surgeries to treat blepharospasm, fronto-glabellar reconstruction flaps, and endoscopic brow lifts [2-4].

The supraorbital nerve has been implicated in cervicogenic headache and migraine. Thus, supraorbital nerve blockade is often used to treat these problems $[5,6]$. Peripheral supraorbital nerve stimulation is sometimes used to treat trigeminal neuralgia. Hence, precise assessment of nerve damage and nerve function is crucial in determining the extent of damage and potential for recovery of the supraorbital nerve. Furthermore, well-established supraorbital nerve conduction techniques can be beneficial to research in the near future.

There are ongoing attempts to establish recording methods in other peripheral nerves $[7,8]$. In the case of the supraorbital nerve, Raffaele et al. [9] suggested a method for measuring conduction in the ophthalmic branch of the trigeminal nerve using orthodromic methods in 1987. Therimadasamy et al. [10] established a method for obtaining antidromic sensory nerve action potentials (SNAPs) in 2011. Other than these two studies $[11,12]$, there has been no electrodiagnostic study providing useful information on the diagnosis of supraorbital neuropathy. Herein, we describe a method for evaluating supraorbital sensory nerve conduction, and we also report two cases of forehead hypoesthesia caused by trauma in which supraorbital nerve injury was diagnosed using this method.

\section{MATERIALS AND METHODS}

This study was approved by the Korea University Anam Hospital Institutional Review Board in 2015. All the subjects were recruited prospectively.

\section{Healthy subjects}

A total of 31 healthy subjects who had no history of trauma or neurological deficits were recruited. Volunteers with diabetes mellitus, chronic alcohol consumption, or other diseases that could affect peripheral nerve were excluded. Subjects were currently not taking any medications and had not had previous surgeries or procedures around their foreheads. The study group was composed of 12 men and 19 women, with a mean ( \pm standard deviation) age of $39.7( \pm 15.6)$ years.

A nerve conduction study was carried out using a Dantec Counterpoint MK2 device (Medtronic, Minneapolis, MN, USA). Bilateral assessments were taken of the foreheads of 29 subjects. Two subjects refused to take part in the bilateral side, so only right side assessment was done. Thus, 60 supraorbital SNAPs were measured in total. Supraorbital SNAPs were recorded using orthodromic and antidromic techniques. For the orthodromic technique, the recording bar electrode was attached immediately superior to the supraorbital notch (Fig. 1). The position of the stimulating electrode was moved incrementally between 3 to $7 \mathrm{~cm}$ laterally from the midline at the level of the hair line. The point evoking the orthodromic SNAP with the largest amplitude was selected. Averaging technique was used to confirm reproducibility and to avoid artifacts. The location of the stimulation site was recorded by measuring the vertical distance from the nasion and the horizontal distance from the midline. When that point was determined, the recording and stimulating electrodes were switched, and the antidromic sensory conduction study was performed (Fig. 2). The stimulating electrode was located at the supraorbital notch, and

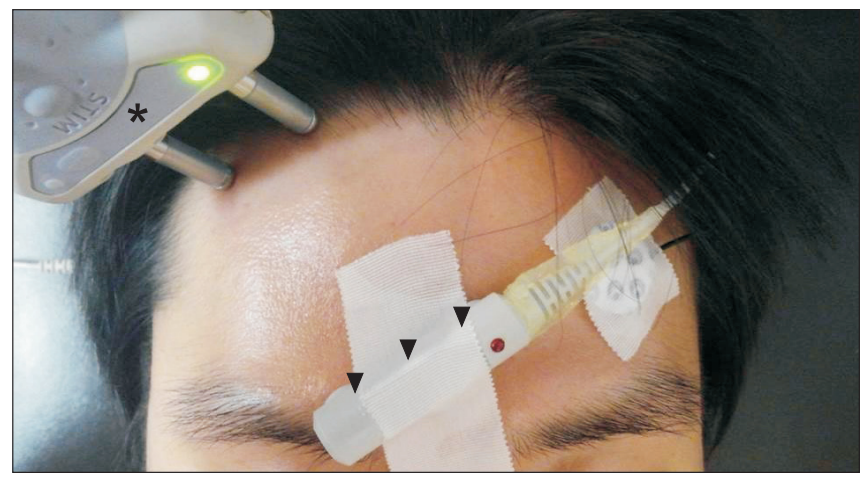

Fig. 1. The orthodromic supraorbital sensory nerve conduction technique showing the recording electrode (arrowhead) and the stimulating electrode (asterisks). 
(A)

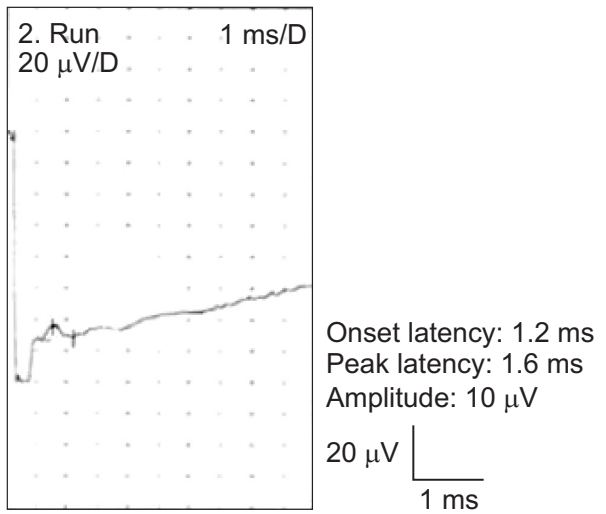

(B)

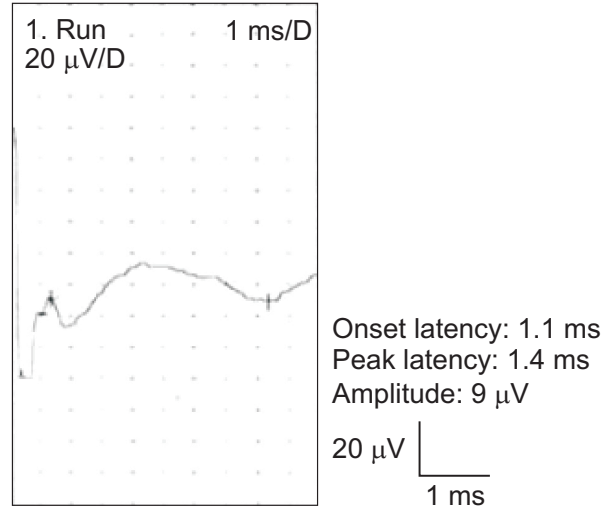

Fig. 2. Supraorbital sensory nerve action potentials obtained using the orthodromic (A) and antidromic (B) techniques in the same subject.

the recording electrode was located at the site where the stimulating electrode had been placed in the orthodromic study. If an appropriate response was not obtainable after 50 or more stimulations, we interpreted the case as having no evoked potential. Stimulation intensity was between 3 and $8 \mathrm{~mA}$. The measured parameters were onset latency, peak latency, and baseline to peak amplitude of the SNAPs.

All statistical analyses were performed using SPSS Statistics ver. 2.0 software (IBM, Armonk, NY, USA). A paired t-test was performed to compare the parameters of SNAP between the sides of the forehead and between the two techniques. The $\mathrm{p}$-values under 0.05 were considered statistically significant. The sample size that would result in statistical significance was estimated using $G^{*}$ Power ver. 3.1.9.2 software (Heinrich-Heine-Universität Düsseldorf, Germany).

\section{Patients}

To evaluate the clinical utility of the new method, supraorbital SNAPs were recorded in two patients with forehead trauma.

\section{Patient 1}

Patient 1 was a 40 -year-old woman with left forehead trauma. She had a laceration on her forehead beause she had fallen down and hit a door 3 months prior to the study. She complained of hypoesthesia in the sensory territory of the left supraorbital nerve (the left forehead and anterior scalp) (Fig. 3A). Although there was no sensation initially, it gradually returned over time, reaching almost $90 \%$ of the normal levels.

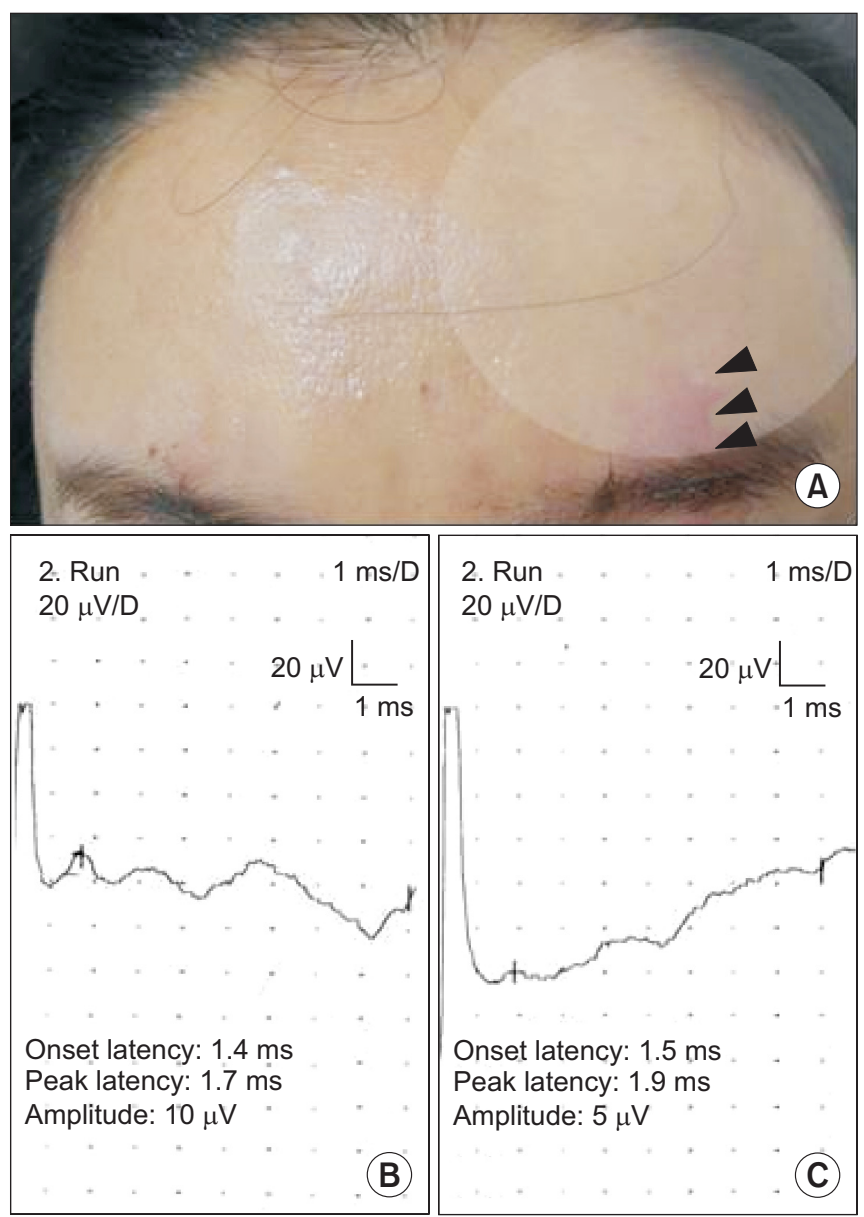

Fig. 3. (A) The patient who had an injury on her left eyebrow and forehead (arrowheads). Hypoesthesia was present on her left forehead and left frontal scalp. (B) Sensory nerve action potential (SNAP) of the right supraorbital nerve obtained using the orthodromic technique. (C) SNAP of the left supraorbital nerve obtained using the orthodromic technique. 


\section{Patient 2}

Patient 2 was a 43-year-old man with a right head laceration. He had fallen off of a bicycle and got a laceration on his midline to right forehead 6 months prior to the study. He complained of paresthesia in his right forehead and scalp. The paresthesia was in the upper region of the scar (Fig. 4A). The paresthesia remained unchanged up to the time of the study.
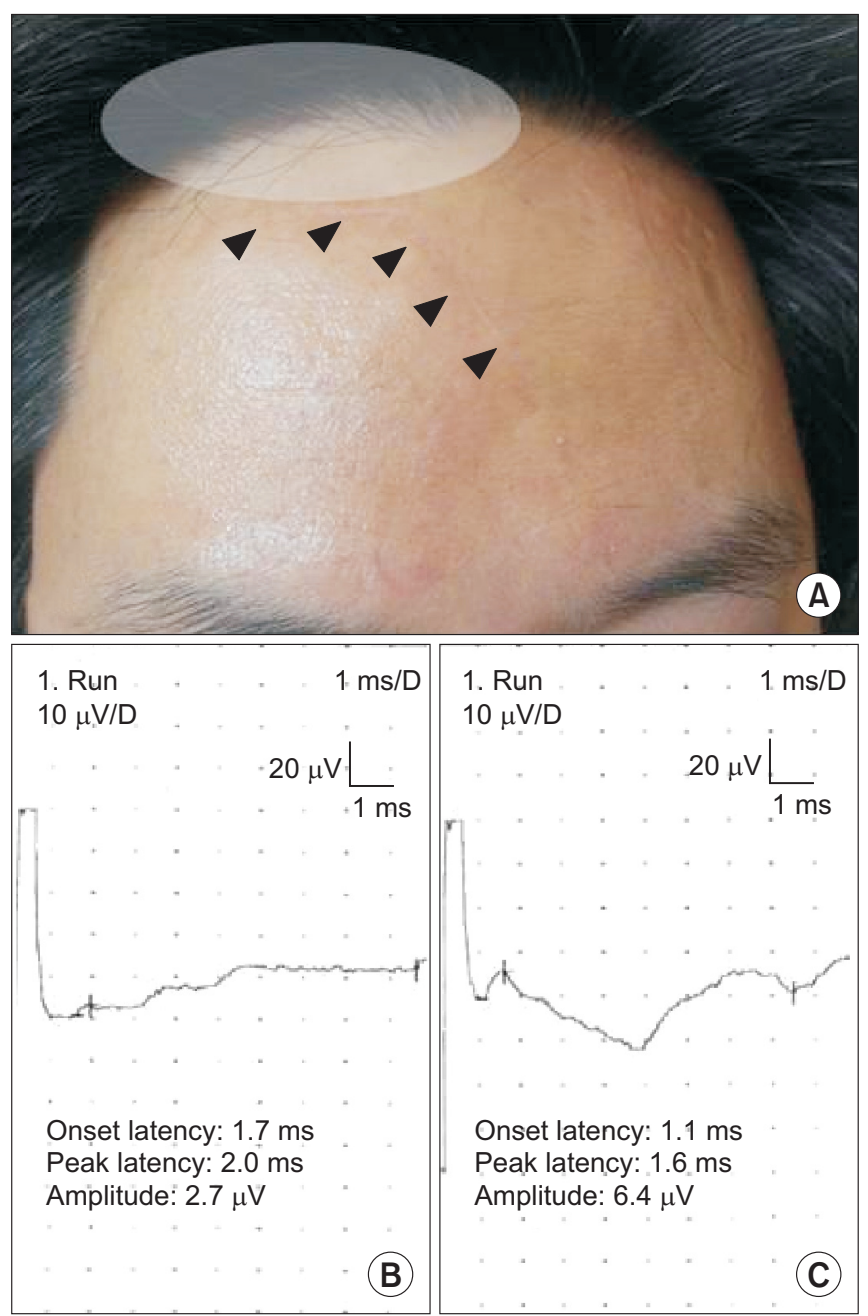

Fig. 4. (A) The patient who had an injury on his right forehead (arrowheads). He experienced paresthesia on his right forehead, on the upper side of the scar. (B) Sensory nerve action potential (SNAP) of the right supraorbital nerve obtained using the orthodromic technique. (C) SNAP of the left supraorbital nerve obtained using the orthodromic technique.

\section{RESULTS}

\section{Healthy subjects}

Thirty-one subjects completed the supraorbital conduction study. The mean age of the subjects was 39.74 \pm 15.57 years (range, 25-73 years). The SNAP response was obtained bilaterally in 29 of the 31 subjects, and unilaterally in 2 subjects. An appropriate biphasic response was elicited in 60 orthodromic trials. In the antidromic study, 53 antidromic SNAPs were obtained. Antidromic SNAPs were not obtained in 7 subjects in whom an orthodromic response was elicited.

The stimulation site was $6.85 \pm 1.08 \mathrm{~cm}$ superior to the nasion and $5.07 \pm 0.77 \mathrm{~cm}$ lateral from the midline. The parameters of orthodromically recorded SNAPs were as follows: onset latency $1.21 \pm 0.22 \mathrm{~ms}$ (range, 0.9-1.6 $\mathrm{ms}$ ), peak latency $1.54 \pm 0.23 \mathrm{~ms}$ (range, 1.2-2.2 ms), and baseline to peak amplitude $4.16 \pm 1.92 \mu \mathrm{V}$ (range, 1.4-10 $\mu \mathrm{V})$. Those of antidromically recorded SNAPs were onset latency $1.31 \pm 0.27 \mathrm{~ms}$ (range, 0.8-1.7 ms), peak latency $1.62 \pm 0.29 \mathrm{~ms}$ (range, 1.3-2.2 ms), and baseline to peak amplitude $4.00 \pm 1.89 \mu \mathrm{V}$ (range, 1.5-9.0 $\mu \mathrm{V}$ ) (Table 1). There was no statistical difference in onset latency, peak latency, or baseline to peak amplitude between the responses obtained using the orthodromic and antidromic methods and no statistical difference between the sides of the forehead. Between-side difference in amplitude was less than $50 \%$ in every subject. The electrophysiologic parameters revealed no differences acoording to gender and age.

Patients

\section{Patient 1}

The supraorbital SNAPs obtained from Patient 1 are

Table 1. Parameters of supraorbital sensory nerve action potentials

\begin{tabular}{lccc}
\hline & $\begin{array}{c}\text { Onset } \\
\text { latency }(\mathrm{ms})\end{array}$ & $\begin{array}{c}\text { Peak } \\
\text { latency }(\mathbf{m s})\end{array}$ & $\begin{array}{c}\text { Amplitude } \\
(\boldsymbol{\mu V})\end{array}$ \\
\hline Orthodromic & $1.21 \pm 0.22^{\mathrm{a})}$ & $1.54 \pm 0.23^{\mathrm{a})}$ & $4.16 \pm 1.92^{\mathrm{a})}$ \\
Antidromic & $1.31 \pm 0.27$ & $1.62 \pm 0.29$ & $4.00 \pm 1.89$ \\
Right side & $1.25 \pm 0.26^{\mathrm{b})}$ & $1.57 \pm 0.26^{\mathrm{b})}$ & $4.16 \pm 2.07^{\mathrm{b})}$ \\
Left side & $1.25 \pm 0.24$ & $1.58 \pm 0.26$ & $4.02 \pm 1.72$ \\
\hline
\end{tabular}

Values are presented as mean \pm standard deviation.

${ }^{a)} p>0.05$ comparison with antidromic method.

${ }^{b)} p>0.05$ comparison with left side. 
shown in Fig. 3B and C. The amplitudes of the supraorbital SNAPs were $5 \mu \mathrm{V}$ on the left and $10 \mu \mathrm{V}$ on the right. The patient was diagnosed with incomplete supraorbital neuropathy given the between-side difference in amplitude.

\section{Patient 2}

The supraorbital SNAPs obtained from Patient 2 are shown in Fig. 4B and C. Supraorbital neuropathy was identified by latency delay and a side-to-side difference in amplitude. The right side had a 2.0-ms peak latency and a $2.7-\mu \mathrm{V}$ amplitude, whereas the left side had a 1.6-ms peak latency and a $6.4-\mu \mathrm{V}$ amplitude.

\section{DISCUSSION}

In this article we describe a technique for measuring supraorbital nerve conduction. Reliable SNAPs can be obtained with either orthodromic or antidromic methods.

In previous studies, Shahani [13] and Jandolo et al. [14] used the blink reflex to evaluate the conduction velocity of the supraorbital nerve. Most past studies measured conduction in the supraorbital nerve indirectly with the blink reflex $[15,16]$. However, use of the blink reflex has limitations. In 1987, Raffaele et al. [9] suggested a method for recording the ophthalmic branch of the trigeminal nerve using an orthodromic method. Raffaele's method was similar to ours. They placed the recording electrode over the supraorbital ridge and the stimulating electrode on the lateral forehead, but their method was not precisely described. Also, their study included only 10 subjects. The amplitude of the supraorbital nerve SNAP was $32.8 \pm 2.8 \mu \mathrm{V}$. Even though they measured peak-topeak amplitude, their observed amplitudes were much larger than those in our study. Considering the fact that the supraorbital nerve is a relatively small sensory nerve fiber, the large amplitude could have resulted from a large volume-conducted positive deflection. Today, it is standard to measure the baseline to peak amplitude; doing so is recommended by the American Association of Neuromuscular \& Electrodiagnostic Medicine (AANEM). Our study reported normal values of supraorbital SNAP amplitude using baseline to peak measurements. In 2012, Therimadasamy et al. [10] recorded antidromic SNAPs from the supraorbital nerve in 17 subjects. They fixed the locations of the stimulating and recording electrodes. Anatomical variations such as size of the forehead, hairline level, scalp, and skin texture of each subject were not considered. Therefore, a consensus regarding an optimal supraorbital nerve conduction method has not been arrived at.

Christensen et al. [17] performed anatomic dissection of 16 cadavers in order to observe the supraorbital nerve. At $2.63 \mathrm{~cm}$ from the midline and $0.25 \mathrm{~cm}$ above the orbital rim, the supraorbital nerve arose from the supraorbital notch and separated into superficial and deep branches. The deep branch, which traveled to the corrugator supercilii and frontalis muscles, ran laterally to the scalp. The superficial branch remained perpendicular to the orbital rim and stayed under the corrugator supercilli and frontalis muscle. It entered the subcutaneous plane by piercing the frontalis muscle at a mean distance of $2.60 \mathrm{~cm}$ above the orbital rim and an average depth of $0.3 \mathrm{~cm}$ [1719]. Hence, we recorded and stimulated the nerve at the hairline level to obtain the maximal SNAPs at the point at which the supraorbital nerve runs most superficially. Supraorbital SNAPs are recorded mostly from the superficial branch because of its anatomic course. Trauma to the forehead can injure the superficial branch of the supraorbital nerve and can cause numbness and paresthesia of the anterior margin of the scalp. The superficial branch often gives rise to multiple sub-branches as it travels in the subcorrugator plane [17]. Although we were able to consistently stimulate the superficial branch, it was technically difficult to stimulate multiple branches because of wide variability in the precise location of these branches. Since we could more easily change the stimulator location with the orthodromic method, we recorded orthodromic SNAPs more easily than antidromic SNAPs.

In this study, the antidromic method produced no response in 7 subjects in which orthodromic resopnses were elicited. The fact that SNAP amplitude in the orthodromic study in 7 patients was relatively small may have created technical pitfalls. There may be additional technical limitations caused by varying head and forehead size and differing scalp, hair, and skin texture.

In our study, the orthodromic method was easier and more accurate than the antidromic method. One of the reasons may be that the muscle artifact is more common in the antidromic method. Another possible reason is that the supraorbital notch can be clearly found, so the 
recording electrode can be easily positioned in the orthodromic study but not in the antidromic study.

In general, the antidromic method produces SNAPs with larger amplitudes than does the orthodromic method [20] since the recording electrodes in the former method are closer to subcutaneous neural tissue. Depending on the anatomical position of the supraorbital nerve, antidromic recording electrodes are closer to the subcutaneous neural tissue because the supraorbital nerve pierces the frontalis muscle and runs subcutaneously at the hairline level. Nevertheless, this study demonstrated that the orthodromic method showed slightly larger amplitudes than the antidromic method, even though the difference was not statistically significant. This result could have been caused by a technical limitation, or perhaps the difference in neural tissue depth was too small to make a statistically meaningful electrophysiological difference.

The orthodromic method produces SNAPs much more easily, as we mentioned above. We can minimize artifacts by simply changing the electrode according to the patients' anatomic variablilty. We could get potentials more easily with the orthodromic study when the amplitude of SNAPs is small, as is supported by our results. In contrast, the nerve fiber is closer to the recording electrodes in the antidromic method. Although it is not clear that that difference is meaningful because of the small sample size, further studies may address the anatomical advantages of the antidromic method.

Both Patient 1 and Patient 2 showed a difference in bilateral supraorbital SNAP amplitude. This fact, considered together with the clinical findings, indicates that $\mathrm{Pa}$ tient 1 has a conduction block of the supraorbital nerve. Her scar was not deep enough to directly damage the supraorbital nerve and her symptom, hypoesthesia, gradually got better until the point that her sensation is about $90 \%$ of normal now. As it is thought that electrophysiologic improvement is evident later than symptomatic improvement, restoring of the amplitude of her supraorbital SNAP is expected.

Patient 2 complained of paresthesia above his scar. The symptom remained unchanged for 6 months after the trauma. The laceration was close to the hairline level, where the supraorbital nerve runs subcutaneously. The laceration could have caused the right supraorbital nerve injury, so the amplitude was low and latency was de- layed.

This study has some limitations. We conducted 60 orthodromic and 53 antidromic trials, but the sample size is still relatively small to clarify the statistical difference between the antidromic and orthodromic group. We estimated sample size to see the difference of amplitude between the two techniques using $\mathrm{G}^{*}$ Power ver. 3.1.9.2 for Windows, and the estimated smaple size was 957 . Since the difference of the amplitude between the two groups is very small, a relatively large number of subjects are needed to create sufficient statistical power. It remains to be proven if these results will generalize to a larger cohort.

In conclusion, in this study we have shown that supraorbital sensory conduction is helpful in confirming a diagnosis of supraorbital neuropathy. Based on the results of this study, the orthodromic technique is recommended, as the responses are better than with the antidromic technique. The orthodromic method is a reliable way to investigate supraorbital nerve conduction, which may be useful in studying some focal and systemic pathologies. Further studies are needed to test the diagnostic utility in patients with supraorbital neuropathy.

\section{CONFLICT OF INTEREST}

No potential conflict of interest relevant to this article was reported.

\section{REFERENCES}

1. Penas-Prado M, Martinez-Salio A, Porta-Etessam J, Villarejo-Galende A, Garcia-Ramos R, Saiz-Diaz RA, et al. Post-traumatic supraorbital neuralgia: a benign condition. Rev Neurol 2007;44:89-91.

2. Knize DM. A study of the supraorbital nerve. Plast Reconstr Surg 1995;96:564-9.

3. Erdogmus S, Govsa F. Anatomy of the supraorbital region and the evaluation of it for the reconstruction of facial defects. J Craniofac Surg 2007;18:104-12.

4. Benvenuti D. Endoscopic brow lifts with injury to the supraorbital nerve and neuroma formation. Plast Reconstr Surg 1999;104:297-8.

5. Bovim G, Sand T. Cervicogenic headache, migraine without aura and tension-type headache: diagnostic blockade of greater occipital and supra-orbital nerves. Pain 1992;51:43-8. 
6. Caputi CA, Firetto V. Therapeutic blockade of greater occipital and supraorbital nerves in migraine patients. Headache 1997;37:174-9.

7. Hemmi S, Kurokawa K, Nagai T, Murakami T, Sunada Y. A novel method to measure sensory nerve conduction of the supraclavicular nerve. Muscle Nerve 2014;50:1005-7.

8. Marchini C, Zambito Marsala S, Fabris F, Fornasier A, Ferracci F. Peroneal nerve orthodromic sensory conduction technique: normative data. Neurol Sci 2009;30:201-5.

9. Raffaele R, Emery P, Palmeri A, Ricca G, Perciavalle V. Sensory nerve conduction velocity of the trigeminal nerve. Electromyogr Clin Neurophysiol 1987;27:115-7.

10. Therimadasamy A, Wilder-Smith EP, Lim AY, Yap YL, Yeo M, Naidu S, et al. Supraorbital nerve conduction study in normal subjects. Muscle Nerve 2012;45:603-4.

11. Oh SJ. Clinical electromyography: nerve conduction studies. 3rd ed. Philadelphia: Lippincott Williams \& Wilkins; 2003.

12. Liveson JA, Ma DM. Laboratory reference for clinical neurophysiology. Philadelphia: F. A. Davis; 1992.

13. Shahani B. The human blink reflex. J Neurol Neurosurg Psychiatry 1970;33:792-800.
14. Jandolo B, Gessini L, Pietrangeli A. Conduction velocity in the human supraorbital nerve. Eur Neurol 1981;20:421-3.

15. Aminoff MJ. Electromyography in clinical practice. Menlo Park: Addison-Wesley; 1978.

16. Kimura J. Electrodiagnosis in diseases of nerve and muscle: principles and practice. 2 nd ed. Philadelphia: F. A. Davis; 1989.

17. Christensen KN, Lachman N, Pawlina W, Baum CL. Cutaneous depth of the supraorbital nerve: a cadaveric anatomic study with clinical applications to dermatology. Dermatol Surg 2014;40:1342-8.

18. Cuzalina AL, Holmes JD. A simple and reliable landmark for identification of the supraorbital nerve in surgery of the forehead: an in vivo anatomical study. J Oral Maxillofac Surg 2005;63:25-7.

19. Beer GM, Putz R, Mager K, Schumacher M, Keil W. Variations of the frontal exit of the supraorbital nerve: an anatomic study. Plast Reconstr Surg 1998;102:33441.

20. Dumitru D, Amato AA, Zwarts MJ. Electrodiagnostic medicine. 2nd ed. Philadelphia: Hanley \& Belfus; 2002. 